19

\title{
Аналитическое описание переходных тепловых процессов в гармонических кристаллах
}

\author{
(c) В.А. Кузькин, А.М. Кривцов
}

Санкт-Петербургский политехнический университет Петра Великого, Санкт-Петербург, Россия

Институт проблем машиноведения РАН,

Санкт-Петербург, Россия

E-mail: kuzkinva@gmail.com

(Поступила в Редакцию 14 июня 2016 г.

В окончательной редакции 20 октября 2016 г.)

Рассматриваются два переходных тепловых процесса, происходящие в однородно нагретых гармонических кристаллах: 1) выравнивание кинетической и потенциальной энергий; 2) перераспределение кинетической энергии по пространственным направлениям. Выведены уравнения, описывающие оба процесса в двухмерном и трехмерном случаях. Получены аналитические решения данных уравнений для квадратной и треугольной решеток. Показано, что характерное время переходных процессов составляет величину порядка десяти периодов колебаний атомов. При переходе разность между кинетической и потенциальной энергиями совершает затухающие колебания. В треугольной решетке амплитуда этих колебаний убывает обратно пропорционально времени, в квадратной - обратно пропорционально корню из времени. Кинетическая энергия в общем случае неравномерно распределяется по пространственным направлениям. Иными словами, кинетическая температура проявляет тензорные свойства. Кроме того, корреляции скоростей различных частиц, вообще говоря, отличны от нуля. Аналитические выкладки подкреплены результатами численного моделирования. Показано, что полученные решения на малых временах хорошо описывают переходные тепловые процессы в слабо нелинейных кристаллах.

Работа выполнена при поддержке Российского научного фонда (гранты № 14-11-00599 и 15-11-00017).

DOI: 10.21883/FTT.2017.05.44396.240

\section{1. Введение}

Количественное описание неравновесных тепловых процессов в кристаллах является одной из актуальных проблем современной физики и имеет особую значимость в связи с развитием нанотехнологий [1-3]. В частности, большой интерес представляют процессы, происходящие в твердом теле при переходе к состоянию термодинамического равновесия $[4,5]$. Неравновесное состояние может быть вызвано, например, прохождением ударных волн [6-9] или быстрым лазерным воздействием [10-14]. В таких случаях кинетические энергии теплового движения атомов в разных направлениях могут существенно различаться [7-9]. Иными словами, кинетическая температура может проявлять тензорные свойства. ${ }^{1}$ Кроме того, могут различаться кинетическая и потенциальная энергии теплового движения атомов. Компьютерное моделирование [15] показывает, что при переходе к состоянию равновесия реализуются два процесса: 1) выравнивание кинетической и потенциальной энергий; 2) перераспределение кинетической энергии по пространственным направлениям. ${ }^{2}$ Настоящая работа

\footnotetext{
${ }^{1}$ В частности, на фронте ударной волны, распространяющейся вдоль оси $x$, выполняются соотношения $T_{x x}>T_{y y}$, где $k_{\mathrm{B}} T_{x x}=m\left\langle v_{x}^{2}\right\rangle$, $k_{\mathrm{B}} T_{y y}=m\left\langle v_{y}^{2}\right\rangle, k_{\mathrm{B}}-$ постоянная Больцмана.

2 Речь идет об изменении энергий, соответствующих движениям частиц в различных направлениях, а не об обмене энергией между
}

посвящена аналитическому описанию данных переходных тепловых процессов в гармонических кристаллах.

Гармонический кристалл представляет собой кристаллическую решетку, состоящую из материальных точек, взаимодействующих посредством линеаризованных (гармонических $)^{3}$ сил. Точки совершают линейные колебания. Такая математическая модель часто используется в литературе для описания тепловых процессов в твердых телах [16-24]. В принципе уравнения динамики гармонического кристалла могут быть решены аналитически. Однако при таком подходе в силу случайности ${ }^{4}$ начальных условий решение получается стохастическим.

При описании тепловых процессов наибольший интерес представляют не случайные движения отдельных частиц, а изменение статистических характеристик, таких как кинетическая температура или ее обобщение тензорная температура [7,9]. Сформулировать задачу для статистических характеристик позволяет корреляционный анализ [16-19]. В рамках данного подхода рассматривается бесконечное множество реализаций кристалла, различающихся только случайными начальными условиями. С использованием осреднения по реализациям

нормальными модами. Последний процесс в гармонических кристаллах отсутствует.

${ }^{3}$ Силы взаимодействия линейно зависят от перемещений частиц.

${ }^{4}$ В тепловых задачах, как правило, частицы имеют случайные начальные скорости. 
выводятся ковариации (математические ожидания от произведений) перемещений и скоростей частиц, для которых получается замкнутая система уравнений. Начальные условия для ковариаций являются детерминированными. Решение системы позволяет, в частности, описать изменение кинетической температуры. Важно отметить, что при выводе уравнений для ковариаций не используется никаких предположений о функции распределения. Вопросы, связанные с поведением функции распределения при переходе к равновесию, обсуждаются, например, в работах [20-22].

Корреляционный анализ применялся для описания тепловых процессов в гармонических кристаллах в работах [16-19]. Основное внимание уделялось процессу распространения тепла. В пионерской работе [16] было получено аналитическое решение стационарной задачи о распространении тепла в гармонической цепочке. Решение показало, что данный процесс не описывается законом Фурье. Исследованию данной аномалии в гармонических и ангармонических кристаллах посвящены, например, работы [19,24-27]. В частности, в работе [19] получено уравнение, описывающее распространение тепла в одномерном гармоническом кристалле.

Корреляционный анализ также позволяет описывать переходные процессы, происходящие в однородно нагретых кристаллах $[17,28]$. В работе [17] рассматривалась гармоническая цепочка со случайными начальными скоростями и нулевыми начальными перемещениями. При этом в начальный момент времени полная энергия системы равна кинетической, а потенциальная энергия равна нулю. Было показано, что со временем кинетическая и потенциальная энергии выравниваются, а их разность (лагранжиан) совершает затухающие колебания, описываемые функцией Бесселя первого рода. Обобщение на случай цепочки на упругом основании приведено в работе [28].

Настоящая работа посвящена аналитическому описанию переходных тепловых процессов в двухмерных и трехмерных однородно нагретых гармонических кристаллах. В начальный момент времени частицы имеют случайные скорости и перемещения. На основе корреляционного анализа выводятся детерминированные уравнения, описывающие два переходных процесса: 1) выравнивание кинетической и потенциальной энергий; 2) перераспределение кинетической энергии по пространственным направлениям. Получаются точные аналитические решения данных уравнений для квадратной и треугольной решеток. Для проверки аналитических выкладок проводится сравнение с численным решением уравнений динамики решетки. Исследуется влияние малой нелинейности на полученные результаты.

Результаты, полученные в работе, позволяют, в частности, оценить характерные времена и частоты переходных тепловых процессов в гармонических кристаллах, а также определить условия, при которых температура проявляет тензорные свойства.
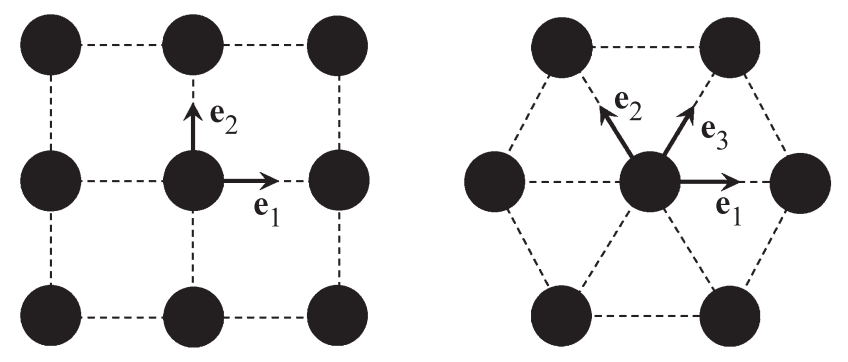

Рис. 1. Векторы $\mathbf{e}_{\alpha}$ для квадратной и треугольной решеток.

\section{2. Уравнения динамики кристалла (стохастическая задача)}

Рассмотрим бесконечную простую кристаллическую решетку, состоящую из одинаковых частиц, соединенных линейными пружинками. Для идентификации частиц будем использовать их радиус-векторы в недеформированном состоянии. Уравнение движения частицы с радиусвектором $\mathbf{r}$ имеет вид

$$
\dot{\mathbf{v}}(\mathbf{r})=\omega_{*}^{2} \sum_{\alpha} \mathbf{e}_{\alpha} \mathbf{e}_{\alpha} \cdot\left(\mathbf{u}\left(\mathbf{r}+\mathbf{a}_{\alpha}\right)-2 \mathbf{u}(\mathbf{r})+\mathbf{u}\left(\mathbf{r}-\mathbf{a}_{\alpha}\right)\right),
$$

где $\mathbf{u}(\mathbf{r}), \mathbf{v}(\mathbf{r})$ - перемещение и скорость частицы с радиус-вектором $\mathbf{r} ; \mathbf{a}_{\alpha}-$ вектор, соединяющий две соседние частицы; $\mathbf{e}_{\alpha}=\mathbf{a}_{\alpha} /\left|\mathbf{a}_{\alpha}\right| ; \omega_{*}=\sqrt{C / m} ; C-$ жесткость связи; $m$ - масса частицы. Суммирование ведется по неколлинеарным направлениям связей $\alpha$. В частности, в квадратной решетке $\alpha=1,2$, в треугольной решетке $\alpha=1,2,3$ (рис. 1).

Далее будем использовать следующую операторную запись уравнений движения:

$$
\begin{gathered}
\dot{\mathbf{v}}(\mathbf{r})=\mathscr{D} \cdot \mathbf{u}(\mathbf{r}), \quad \mathscr{D}=\omega_{*}^{2} \sum_{\alpha} \mathbf{e}_{\alpha} \mathbf{e}_{\alpha} \Delta_{\alpha}^{2}, \\
\Delta_{\alpha}^{2} \mathbf{u}(\mathbf{r})=\mathbf{u}\left(\mathbf{r}+\mathbf{a}_{\alpha}\right)-2 \mathbf{u}(\mathbf{r})+\mathbf{u}\left(\mathbf{r}-\mathbf{a}_{\alpha}\right) .
\end{gathered}
$$

Здесь $\mathscr{D}$ - тензорный разностный оператор второго порядка. 5 В начальный момент времени перемещения и скорости частиц имеют вид

$$
\mathbf{u}(\mathbf{r})=\mathbf{u}_{0}(\mathbf{r}), \mathbf{v}(\mathbf{r})=\mathbf{v}_{0}(\mathbf{r}),
$$

где $\mathbf{u}_{0}, \mathbf{v}_{0}$ - независимые случайные векторы с нулевым математическим ожиданием. Здесь и далее индекс 0 обозначает начальные условия.

Первое из уравнений (2) с начальными условиями (3) полностью определяет динамику кристалла. В принципе оно может быть решено аналитически. При начальных условиях (3) получающиеся в результате решения перемещения и скорости частиц являются случайными величинами. Исследование характеристик движения отдельных частиц важно, например, при описании разрушения [29,30]. При описании тепловых процессов

\footnotetext{
5 Таким образом, движение частиц описывается дифференциальноразностным уравнением.
} 
больший интерес представляют не случайные движения отдельных частиц, а изменение статистических характеристик. В качестве таких характеристик могут выступать ковариации (математические ожидания от произведения) скоростей и перемещений частиц. Начальные условия для ковариаций являются детерминированными. Замкнутая система уравнений, описывающих динамику ковариаций, выводится в следующем разделе.

\section{3. Уравнения динамики ковариаций (детерминированная задача)}

Рассмотрим бесконечное множество реализаций одного и того же кристалла. Реализации различаются значениями скоростей и перемещений частиц, являющимися случайными величинами (см. формулу (3)). Рассмотрение множества реализаций позволяет ввести ковариации перемещений и скоростей, определенные для пары частиц $i, j$ следующими формулами:

$$
\xi\left(\mathbf{r}_{i}, \mathbf{r}_{j}\right)=\left\langle\mathbf{u}_{i} \mathbf{u}_{j}\right\rangle, \kappa\left(\mathbf{r}_{i}, \mathbf{r}_{j}\right)=\left\langle\mathbf{v}_{i} \mathbf{v}_{j}\right\rangle, \quad v\left(\mathbf{r}_{i}, \mathbf{r}_{j}\right)=\left\langle\mathbf{u}_{i} \mathbf{v}_{j}\right\rangle,
$$

где $\mathbf{r}_{i}$ и $\mathbf{r}_{j}$ - радиус-векторы соответствующих частиц; $\mathbf{u}_{i}=\mathbf{u}\left(\mathbf{r}_{i}\right) ; \mathbf{v}_{i}=\mathbf{v}\left(\mathbf{r}_{i}\right)$. Здесь и далее угловые скобки обозначают математическое ожидание. При этом считается, что перемещения и скорости являются центрированными случайными величинами: $\left\langle\mathbf{u}_{i}\right\rangle=0,\left\langle\mathbf{v}_{i}\right\rangle=0$.

Будем рассматривать процессы, происходящие в однородно нагретом кристалле. В таком случае выполняются соотношения

$$
\begin{gathered}
\xi\left(\mathbf{r}_{i}, \mathbf{r}_{j}\right)=\xi\left(\mathbf{r}_{i}-\mathbf{r}_{j}\right), \kappa\left(\mathbf{r}_{i}, \mathbf{r}_{j}\right)=\kappa\left(\mathbf{r}_{i}-\mathbf{r}_{j}\right), \\
v\left(\mathbf{r}_{i}, \mathbf{r}_{j}\right)=v\left(\mathbf{r}_{i}-\mathbf{r}_{j}\right) .
\end{gathered}
$$

Далее для краткости аргумент $\mathbf{r}_{i}-\mathbf{r}_{j}$, как правило, будет опущен. Важно отметить, что множество точек, задаваемых векторами $\mathbf{r}_{i}-\mathbf{r}_{j}$, образует ту же решетку, что и частицы кристалла. Поэтому задачи для ковариаций решаются „на той же решетке“, что и уравнения движения (2).

Дифференцируя ковариации (4) по времени с учетом уравнений движения (2) и соотношений (5), получим следующие уравнения динамики:

систему уравнений первого порядка

$$
\begin{gathered}
\dot{\xi}=v+v^{*}, \dot{\kappa}=\mathscr{D} \cdot v+v^{*} \cdot \mathscr{D}, \dot{v}=\kappa+\xi \cdot \mathscr{D}, \\
\left(v\left(\mathbf{r}_{i}-\mathbf{r}_{j}\right)\right)^{*}=v^{T}\left(\mathbf{r}_{j}-\mathbf{r}_{i}\right)
\end{gathered}
$$

систему уравнений второго порядка для $\xi$ и $\kappa$

$\ddot{\xi}=\mathscr{D} \cdot \xi+\xi \cdot \mathscr{D}+2 \kappa, \ddot{\kappa}=\mathscr{D} \cdot \kappa+\kappa \cdot \mathscr{D}+2 \mathscr{D} \cdot \xi \cdot \mathscr{D} ;$

систему уравнений второго порядка для $v$

$\ddot{v}=\mathscr{D} \cdot v+\left(v+2 v^{*}\right) \cdot \mathscr{D}, \ddot{v}^{*}=\mathscr{D} \cdot\left(v^{*}+2 v\right)+v^{*} \cdot \mathscr{D} ;$ уравнение четвертого порядка

$$
\dddot{\kappa}-2(\mathscr{D} \cdot \ddot{\kappa}+\ddot{\kappa} \cdot \mathscr{D})+\mathscr{D}^{2} \cdot \kappa-2 \mathscr{D} \cdot \kappa \cdot \mathscr{D}+\kappa \cdot \mathscr{D}^{2}=0 .
$$

Здесь $\mathscr{D}^{2}=\mathscr{D} \cdot \mathscr{D} ; v^{T}-$ транспонированный тензор $v$. Отметим, что уравнению (9) также удовлетворяет ковариация перемещений $\xi$. Уравнения (6)-(9) являются замкнутыми и позволяют определить значения всех ковариаций в любой момент времени.

Покажем, что для ковариации скоростей и перемещений $v$ выполняется несколько законов сохранения. Из (6) следует, что

$$
\dot{v}-\dot{v}^{*}=\xi \cdot \mathscr{D}-\mathscr{D} \cdot \xi .
$$

Домножая обе части уравнения (10) на $\mathscr{D}^{n}$, получим 6

$$
\mathscr{D}^{n} \cdots\left(v-v^{*}\right)=\text { const }, \quad n=0,1,2, \ldots
$$

Здесь и далее $\mathscr{D}^{0}=\mathscr{Y}$, где $\mathscr{I}$ - единичный тензор. Из теоремы Гамильтона-Кэли следует, что количество независимых законов сохранения (11) равно размерности пространства.

Начальные условия к уравнениям (6)-(9) однозначно определяются начальными перемещениями и скоростями частиц. В частности, для уравнений (6) имеем начальные условия

$$
\xi=\left\langle\mathbf{u}_{i}^{0} \mathbf{u}_{j}^{0}\right\rangle, \kappa=\left\langle\mathbf{v}_{i}^{0} \mathbf{v}_{j}^{0}\right\rangle, \quad v=\left\langle\mathbf{u}_{i}^{0} \mathbf{v}_{j}^{0}\right\rangle,
$$

где $\mathbf{u}_{i}^{0}, \mathbf{v}_{i}^{0}$ - начальные скорости и перемещения. Важно отметить, что в отличие от начальных условий (3) для перемещений частиц условия (12) для ковариаций являются детерминированными.

Таким образом, рассмотрение множества реализаций и введение ковариаций позволяет перейти от задачи для перемещений со случайными начальными условиями (3) к задаче для ковариаций с детерминированными начальными условиями (12). При этом динамика ковариаций описывается уравнениями (6)-(8) или (9).

\section{4. Обобщенные энергии}

При описании тепловых процессов одной из центральных величин является кинетическая температура $T$ [31] или ее обобщение - тензорная температура $\mathscr{T}[7,9]^{7}$

$$
\frac{k_{\mathrm{B}}}{2} \mathscr{T}\left(\mathbf{r}_{i}\right)=\frac{m}{2}\left\langle\mathbf{v}_{i} \mathbf{v}_{i}\right\rangle=\left.\frac{m}{2} \kappa\right|_{i=j}, \quad T=\frac{1}{d} \operatorname{tr} \mathscr{T},
$$

где $k_{\mathrm{B}}-$ постоянная Больцмана, $d-$ размерность пространства. В предыдущем разделе показано, что для получения замкнутой системы уравнений кинетической температуры недостаточно: необходимо рассмотрение ковариации скоростей различных частиц (см. (9)).

\footnotetext{
6 Двумя точками обозначается двойное скалярное произведение например $\mathbf{a b} \cdot \mathbf{c d}=(\mathbf{b} \cdot \mathbf{c})(\mathbf{a} \cdot \mathbf{d})$.

${ }^{7}$ В данных работах проводилось молекулярно-динамическое моделирование распространения ударных волн в жидкости.
} 
В работах $[17,19,32]$ на примере одномерного кристалла показано, что при описании тепловых процессов удобно рассматривать линейные комбинации ковариаций, имеющие смысл обобщенных энергий. Введем обобщенные (двухчастичные) кинетические $\mathscr{K}$ и потенциальные $\mathscr{U}$ энергии [19], определенные для пары частиц $i, j$ :

$$
\mathscr{K}\left(\mathbf{r}_{i}-\mathbf{r}_{j}\right)=\frac{m}{2} \kappa, \mathscr{U}\left(\mathbf{r}_{i}-\mathbf{r}_{j}\right)=-\frac{m}{4}(\mathscr{D} \cdot \xi+\xi \cdot \mathscr{D}) .
$$

При $i=j$ следы тензоров $\mathscr{K}, \mathscr{U}$ соответствуют обычным кинетической и потенциальной энергиям, приходящимся на частицу. Далее для краткости аргумент обобщенных энергий $\mathbf{r}_{i}-\mathbf{r}_{j}$, как правило, будет опущен. Введем также обобщенную полную энергию $\mathscr{H}$ и обобщенный лагранжиан $\mathscr{L}$ :

$$
\mathscr{H}=\mathscr{K}+\mathscr{U}, \quad \mathscr{L}=\mathscr{K}-\mathscr{U} .
$$

В предыдущем разделе показано, что ковариации $\xi$ и $\kappa$ удовлетворяют уравнению (9). Следовательно, уравнению (9) удовлетворяют и обобщенные энергии $\mathscr{K}, \mathscr{U}, \mathscr{L}, \mathscr{H}$. Далее для краткости будем пользоваться операторной записью данного уравнения

$$
\mathfrak{L} \mathscr{G}=0,
$$

где

$$
\begin{gathered}
\mathscr{G}=\{\xi, \kappa, \mathscr{K}, \mathscr{U}, \mathscr{L}, \mathscr{H}\}, \\
\mathfrak{L} \mathscr{G}=\dddot{\mathscr{G}}-2(\mathscr{D} \cdot \ddot{\mathscr{G}}+\ddot{\mathscr{G}} \cdot \mathscr{D})+\mathscr{D}^{2} \cdot \mathscr{G} \\
-2 \mathscr{D} \cdot \mathscr{G} \cdot \mathscr{D}+\mathscr{G} \cdot \mathscr{D}^{2},
\end{gathered}
$$

где $\mathfrak{L}$ - линейный дифференциально-разностный оператор. Отметим, что уравнению (16) удовлетворяют симметричные и антисимметричные части тензоров $\xi, \kappa, \mathscr{K}, \mathscr{U}, \mathscr{L}, \mathscr{H}^{8}$

В результате получаем, что ковариации и обобщенные энергии удовлетворяют уравнению (16). Начальные условия к уравнению (16) однозначно определяются начальными перемещениями и скоростями частиц (3).

Покажем, что для обобщенной полной энергии $\mathscr{H}$ выполняется несколько законов сохранения, аналогичных (11). Из системы уравнений (6) следует соотношение

$$
\dot{\mathscr{H}}=\frac{m}{4}\left(\mathscr{D} \cdot\left(v-v^{*}\right)-\left(v-v^{*}\right) \cdot \mathscr{D}\right) .
$$

Домножая уравнение (17) на $\mathscr{D}^{n}$, получим законы сохранения

$$
\mathscr{D}^{n} \ldots \mathscr{H}=\text { const }, \quad n=0,1,2, \ldots .
$$

При $n=0$ и $i=j$ формула (18) дает закон сохранения энергии. Из теоремы Гамильтона-Кэли следует, что количество независимых законов (18) равно размерности пространства.

\footnotetext{
${ }^{8}$ Следовательно, симметричные и антисимметричные части обобщенных энергий могут рассматриваться независимо.
}

Таким образом, динамика обобщенных энергий описывается уравнением (16) с детерминированными начальными условиями. Решение данного уравнения для обобщенной кинетической энергии позволяет, в частности, определить поведение тензорной температуры (13). Также отметим, что для обобщенной полной энергии выполняются законы сохранения (18), которые в следующем разделе используются для определения значений обобщенных энергий в стационарном состоянии.

\section{5. Стационарное (равновесное) состояние}

Численное решение уравнений динамики решетки (2) показывает, что после быстрого переходного процесса кристалл приходит в практически стационарное состояние. Здесь и далее стационарным называется состояние кристалла, в котором вторые производные по времени от ковариаций равны нулю. В этом разделе определяется связь значений обобщенных энергий в стационарном состоянии с начальными условиями.

Отбрасывая производные по времени в уравнении (16), записанном для $\mathscr{H}$, и используя законы сохранения (18), получим замкнутую систему уравнений для определения стационарного значения $\mathscr{H}$

$$
\begin{gathered}
\operatorname{tr} \mathscr{H}=\operatorname{tr} \mathscr{H}_{0}, \mathscr{D} \cdots \mathscr{H}=\mathscr{D} \cdots \mathscr{H}_{0}, \mathscr{D}^{2} \cdots \mathscr{H}=\mathscr{D}^{2} \cdots \mathscr{H}_{0}, \\
\mathscr{D}^{2} \cdot \mathscr{H}-2 \mathscr{D} \cdot \mathscr{H} \cdot \mathscr{D}+\mathscr{H} \cdot \mathscr{D}^{2}=0,
\end{gathered}
$$

где $\mathscr{H}_{0}-$ начальное значение обобщенной полной энергии. Первое уравнение системы (19) определяет шаровую часть обобщенной полной энергии, а остальные - девиатор. Данные уравнения могут быть решены аналитически, например, с использованием дискретного преобразования Фурье (см. подраздел 8.2).

Определим соотношение между обобщенными кинетической и потенциальной энергиями $\mathscr{K}$ и $\mathscr{U}$ в стационарном состоянии. Перепишем первое из уравнений (7) в виде

$$
\frac{m}{4} \ddot{\xi}=\mathscr{L}
$$

Отбрасывая производную при переходе к стационарному состоянию, получим

$$
\mathscr{L}=0, \quad \mathscr{K}=\mathscr{U}=\frac{1}{2} \mathscr{H} .
$$

Из формул (21) следует, что при переходе к стационарному состоянию обобщенные кинетическая и потенциальная энергии выравниваются.

Первое уравнение системы (19) и уравнения (21) определяют следы обобщенных энергий

$$
\operatorname{tr} \mathscr{K}=\operatorname{tr} \mathscr{U}=\frac{1}{2} \operatorname{tr} \mathscr{H}_{0} .
$$

При $i=j$ соотношение (22) означает равенство кинетической и потенциальной энергий в стационарном 
состоянии. Данный факт также может быть получен на основе теоремы о вириале [33]. Однако в отличие от подхода, используемого в данной работе, теорема о вириале не описывает переход к стационарному состоянию. Девиаторы обобщенных энергий находятся из решения системы (19). Отметим, что система (19) справедлива как в двухмерном, ${ }^{9}$ так и в трехмерном случае.

Таким образом, в стационарном состоянии обобщенная кинетическая энергия равна обобщенной потенциальной энергии. Их значения связаны с начальными условиями уравнений (19), (21) и (22). Решение данных уравнений для квадратной и треугольной решеток приведено далее. В частности, в подразделе 8.2 показано, что тензор температуры (13), вообще говоря, не является шаровым даже в стационарном состоянии.

\section{6. Переход к стационарному состоянию}

Из полученных выше уравнений видно, что при переходе к стационарному состоянию реализуется два процесса. Во-первых, выравниваются кинетическая и потенциальная энергии. Данный процесс описывается уравнением (16). Во-вторых, может происходить перераспределение энергии по пространственным направлениям. Перераспределение происходит, например, в случае, когда кинетические энергии движений частиц по различным направлениям в начальный момент различаются $\left(\left\langle v_{x}^{2}\right\rangle \neq\left\langle v_{y}^{2}\right\rangle\right)$. В этом разделе рассматривается динамика данного переходного процесса.

Разность энергий, соответствующих различным пространственным направлениям, характеризуется величиной $\operatorname{dev} \mathscr{H}{ }^{10}$ Как было показано ранее, тензор $\mathscr{H}$ удовлетворяет уравнению (16). Из закона сохранения (18) следует, что след $\mathscr{H}$ не зависит от времени. Тогда нетрудно показать, что девиатор $\mathscr{H}$ также удовлетворяет уравнению (16)

$$
\mathfrak{L}(\operatorname{dev} \mathscr{H})=0 .
$$

Если начальные скорости и перемещения частиц заданы независимо $(v=0)$, то начальные условия к уравнению (23) имеют вид

$$
\operatorname{dev} \mathscr{H}=\operatorname{dev} \mathscr{H}_{0}, \operatorname{dev} \dot{\mathscr{H}}=0, \operatorname{dev} \ddot{\mathscr{H}}=0,
$$

$\operatorname{dev} \ddot{\mathscr{H}}=-\frac{m}{4}\left(\mathscr{D}^{2} \cdot \operatorname{dev} \xi_{0}-2 \mathscr{D} \cdot \operatorname{dev} \xi_{0} \cdot \mathscr{D}+\operatorname{dev} \xi_{0} \cdot \mathscr{D}^{2}\right)$,

где $\xi_{0}, \mathscr{H}_{0}-$ начальные значения ковариации перемещений и обобщенной полной энергии. Из (24) следует, что на процесс перераспределения энергии по пространственным направлениям влияют величины $\operatorname{dev} \mathscr{H}_{0}$ и $\operatorname{dev} \xi_{0}$, которые могут быть заданы независимо. Однако

\footnotetext{
${ }^{9}$ В двухмерном случае в силу теоремы Гамильтона-Кэли третье уравнение системы (19) является следствием первых двух.

${ }^{10} \operatorname{dev} \mathscr{H}=\mathscr{H}-\frac{1}{d} \operatorname{tr}(\mathscr{H}) \mathscr{Y}$, где $d-$ размерность пространства, $\mathscr{Y}$ единичный тензор.
}

на значение $\operatorname{dev} \mathscr{H}$ в стационарном состоянии влияет только $\operatorname{dev} \mathscr{H}_{0}$ (см. формулы (19)).

Таким образом, при переходе к стационарному состоянию реализуется два процесса: выравнивание кинетической и потенциальной энергий, а также перераспределение энергии по направлениям. Данные процессы описываются одним уравнением (16), записанным для $\mathscr{L}$ и $\operatorname{dev} \mathscr{H}$ соответственно. Решение уравнения (16) для квадратной и треугольной решеток приведено далее. В частности, для треугольной решетки показано, что при больших временах решение уравнения (16) для $\operatorname{dev} \mathscr{H}$ стремится к решению системы (19), описывающей стационарное состояние.

\section{7. Квадратная решетка}

Рассмотрим бесконечную квадратную решетку с базисными векторами $\mathbf{e}_{1}, \mathbf{e}_{2}$ (рис. 1). Частицы совершают колебания в плоскости решетки. ${ }^{11}$ Данная решетка используется в качестве простейшего примера, позволяющего продемонстрировать идеи, изложенные выше. Пусть в начальный момент времени частицы имеют независимые случайные скорости, а их перемещения равны нулю. При таких начальных условиях тензоры $\mathscr{H}$ и $\mathscr{L}$ являются симметричными. ${ }^{12}$ Рассмотрим процесс перераспределения энергии по пространственным направлениям и процесс выравнивания кинетической и потенциальной энергий.

Ранее было показано, что обобщенные энергии зависят от векторов $\mathbf{r}_{i}-\mathbf{r}_{j}$, соединяющих частицы. Точки, задаваемые данными векторами, образуют квадратную решетку. Будем использовать следующее представление для $\mathbf{r}_{i}-\mathbf{r}_{j}$

$$
\mathbf{r}_{i}-\mathbf{r}_{j}=a\left(k \mathbf{e}_{1}+n \mathbf{e}_{2}\right),
$$

где $k, n$ - целые числа, $a$ - равновесное расстояние.

Рассмотрим динамику обобщенной полной энрегии $\mathscr{H}$. При этом ограничимся случаем, когда компоненты начальных скоростей частиц по направлениям $\mathbf{e}_{1}$ и $\mathbf{e}_{2}$ независимы. Тогда начальные условия для $\mathscr{H}$ имеют вид

$$
\begin{gathered}
\mathscr{H}=\mathscr{K}_{0}=\left(K_{1}^{0} \mathbf{e}_{1} \mathbf{e}_{1}+K_{2}^{0} \mathbf{e}_{2} \mathbf{e}_{2}\right) \delta_{k} \delta_{n}, \\
\dot{\mathscr{H}}=0, \quad \ddot{\mathscr{H}}=0, \quad \dddot{\mathscr{H}}=0,
\end{gathered}
$$

где $\delta_{n}-$ функция, равная единице при $n=0$ и равная нулю во всех остальных случаях. Изменение $\mathscr{H}$ при переходе к стационарному состоянию описывается уравнением (9), где оператор $\mathscr{D}$ имеет вид

$$
\mathscr{D}=\omega_{*}^{2}\left(\Delta_{1}^{2} \mathbf{e}_{1} \mathbf{e}_{1}+\Delta_{2}^{2} \mathbf{e}_{2} \mathbf{e}_{2}\right) .
$$

\footnotetext{
11 Отметим, что в литературе часто рассматривается другой случай, когда частицы совершают движения, перпендикулярные плоскости решетки (см., например, работу [34]).

12 Ранее отмечалось, что антисимметричные части тензоров $\mathscr{H}$ и $\mathscr{L}$ удовлетворяют уравнению (16). В рассматриваемом случае начальные условия для антисимметричных частей будут нулевыми.
} 
С учетом представления (27) уравнение (9) распадается на независимые скалярные уравнения относительно $H_{i j}=\mathbf{e}_{i} \cdot \mathscr{H} \cdot \mathbf{e}_{j}$ :

$$
\begin{array}{r}
\dddot{H}_{11}-4 \omega_{*}^{2} \Delta_{1}^{2} \ddot{H}_{11}=0, \dddot{H}_{22}-4 \omega_{*}^{2} \Delta_{2}^{2} \ddot{H}_{22}=0, \\
\dddot{H}_{12}-2 \omega_{*}^{2}\left(\Delta_{1}^{2}+\Delta_{2}^{2}\right) \ddot{H}_{12}+\omega_{*}^{4}\left(\Delta_{1}^{2}-\Delta_{2}^{2}\right)^{2} H_{12}=0 .
\end{array}
$$

Видно, что компоненты тензора обобщенной полной энергии $\mathscr{H}$ меняются независимо. Следовательно, в квадратной решетке перераспределения энергии по пространственным направлениям не происходит. В частности, при начальных условиях (26) уравнения (28) имеют решение $\mathscr{H}=\mathscr{K}_{0}$, т. е. обобщенная полная энергия сохраняется.

Рассмотрим теперь процесс выравнивания кинетической и потенциальной энергий. Начальные условия для обобщенного лагранжиана $\mathscr{L}$ имеют вид

$$
\mathscr{L}=\mathscr{K}_{0}, \quad \dot{\mathscr{L}}=0, \quad \ddot{\mathscr{L}}=2\left(\mathscr{D} \cdot \mathscr{K}_{0}+\mathscr{K}_{0} \cdot \mathscr{D}\right), \ddot{\mathscr{L}}=0 .
$$

Нетрудно показать, что компоненты $L_{i j}=\mathbf{e}_{i} \cdot \mathscr{L} \cdot \mathbf{e}_{j}$ обобщенного лагранжиана удовлетворяют уравнениям (28). При начальных условиях (29) уравнение для $L_{12}$ имеет тривиальное решение. Уравнения для $L_{11}$ и $L_{22}$ переписываются в виде

$$
\ddot{L}_{11}-4 \omega_{*}^{2} \Delta_{1}^{2} L_{11}=0, \quad \ddot{L}_{22}-4 \omega_{*}^{2} \Delta_{2}^{2} L_{22}=0 .
$$

Уравнения (30) аналогичны уравнению динамики лагранжиана в гармонической цепочке, полученному в работе [17]. Решение уравнений (30) с начальными условиями (29) имеет вид [17]

$$
\mathscr{L}=K_{1}^{0} J_{2 k}\left(4 \omega_{*} t\right) \delta_{n} \mathbf{e}_{1} \mathbf{e}_{1}+K_{2}^{0} J_{2 n}\left(4 \omega_{*} t\right) \delta_{k} \mathbf{e}_{2} \mathbf{e}_{2},
$$

где $J$ - функция Бесселя первого рода. Применяя асимптотические формулы для функции Бесселя, нетрудно показать, что обобщенный лагранжиан совершает затухающие колебания с амплитудой, обратно пропорциональной корню из времени. Учитывая сохранение обобщенной полной энергии $\mathscr{H}=\mathscr{K}_{0}$, в стационарном состоянии имеем

$$
\mathscr{K}=\mathscr{U}=\frac{1}{2} \mathscr{K}_{0}
$$

Из формул (13), (32) видно, что тензор температуры в стационарном состоянии является шаровым, только если начальные скорости равномерно распределены по направлениям (тензор $\mathscr{K}_{0}$ шаровой).

Таким образом, в квадратной решетке кинетические и потенциальные энергии, соответствующие одному направлению, выравниваются. Выравнивание происходит по тому же закону, что и в одномерной цепочке [17]. Перераспределения энергии по направлениям не происходит. Тензор температуры (13), вообще говоря, не является шаровым.

\section{8. Треугольная решетка}

8.1. Общие соотношения. В этом разделе рассматриваются переходные тепловые процессы в треугольной решетке (рис. 1). Как уже отмечалось, обобщенные энергии зависят от векторов $\mathbf{r}_{i}-\mathbf{r}_{j}$. Множество точек, задаваемых векторами $\mathbf{r}_{i}-\mathbf{r}_{j}$, также образует треугольную решетку. Далее все аналитические решения строятся для ячейки периодичности, точки которой нумеруются парой индексов $k, n$

$$
\begin{gathered}
\mathbf{r}_{i}-\mathbf{r}_{j}=a\left(k \mathbf{e}_{1}+n \mathbf{e}_{2}\right), k, n=-N, \ldots, N, \\
\mathbf{e}_{1}=\mathbf{i}, \quad \mathbf{e}_{2}=-\frac{1}{2} \mathbf{i}+\frac{\sqrt{3}}{2} \mathbf{j}, \quad \mathbf{e}_{3}=\mathbf{e}_{1}+\mathbf{e}_{2},
\end{gathered}
$$

где $2 N+1$ - число точек вдоль одной из сторон ячейки периодичности; $\mathbf{i}, \mathbf{j}$ - орты декартового базиса; векторы $\mathbf{e}_{1}, \mathbf{e}_{2}, \mathbf{e}_{3}$ показаны на рис. 1. Ячейка периодичности имеет форму ромба. В точке ромба с индексами $k, n$ заданы обобщенные энергии $\mathscr{K}_{k, n}, \mathscr{U}_{k, n}$. В работе [35] показано, что такая нумерация удобна для аналитического решения задач для треугольной решетки.

Обобщенные энергии определяются из решения разностных уравнений (19) и дифференциально-разностных уравнений (16), (23). Для решения используется дискретное преобразование Фурье. Прямое и обратное дискретные преобразования Фурье, например, для обобщенной полной энергии $\mathscr{H}_{k, n}=\mathscr{H}\left(a\left(k \mathbf{e}_{1}+n \mathbf{e}_{2}\right)\right)$ определяются формулами

$$
\begin{gathered}
\hat{\mathscr{H}}_{s, p}=\sum_{k, n=-N}^{N} \mathscr{H}_{k, n} e^{-2 i\left(\theta_{s} k+\theta_{p} n\right)}, \theta_{s}=\frac{\pi s}{2 N+1}, \\
\mathscr{H}_{k, n}=\frac{1}{(2 N+1)^{2}} \sum_{s, p=-N}^{N} \hat{\mathscr{H}}_{s, p} e^{2 i\left(\theta_{s} k+\theta_{p} n\right)},
\end{gathered}
$$

где $i$ - мнимая единица. Здесь и далее Фурье-образ обозначается „шляпкой“. Далее индексы $k, n$ и $s, p$ у обобщенных энергий и их образов для краткости опущены.

Решения, получаемые с использованием дискретного преобразования Фурье (34), описывают процессы, происходящие в конечных кристаллах при периодических граничных условиях. Случай бесконечного кристалла получается предельным переходом при $N \rightarrow \infty$.

8.2. Стационарное состояние, тензорная температура. Вычислим стационарные значения обобщенных энергий $\mathscr{K}, \mathscr{U}$ и тензорной температуры (13) в треугольной решетке. Их следы определяются формулой (22), а девиаторы выражаются через $\operatorname{dev} \mathscr{H}$

$$
\operatorname{dev} \mathscr{K}=\operatorname{dev} \mathscr{U}=\frac{1}{2} \operatorname{dev} \mathscr{H} .
$$

Следовательно, задача сводится к определению $\operatorname{dev} \mathscr{H}$ из системы разностных уравнений (19). Для решения 
системы (19) будем использовать соотношение

$$
\mathscr{D} \cdot \mathscr{H}=\mathscr{H} \cdot \mathscr{D} .
$$

Нетрудно проверить, что соотношение (36) является, по крайней мере, частным решением последнего из уравнений (19). Сравнение с численным решением уравнений динамики решетки (2) показывает, что формула (36) приводит к корректным результатам.

Представим девиаторы тензоров $\mathscr{H}, \mathscr{H}_{0}$ во введенном декартовом базисе $\mathbf{i}, \mathbf{j}$

$$
\begin{aligned}
\operatorname{dev} \mathscr{H} & =H_{1}(\mathbf{i i}-\mathbf{j} \mathbf{j})+H_{2}(\mathbf{i j}+\mathbf{j i}), \\
\operatorname{dev} \mathscr{H}_{0} & =H_{1}^{0}(\mathbf{i i}-\mathbf{j} \mathbf{j})+H_{2}^{0}(\mathbf{i j}+\mathbf{j i}) .
\end{aligned}
$$

Применим дискретное преобразование Фурье (34) к системе уравнений (19) с учетом формул (36) и (37). Проецируя получившиеся уравнения для образов на $\mathbf{i}, \mathbf{j}$, получим:

$$
\begin{gathered}
A_{s, p}\left(\hat{H}_{1}-\hat{H}_{1}^{0}\right)+B_{s, p}\left(\hat{H}_{2}-\hat{H}_{2}^{0}\right)=0, B_{s, p} \hat{H}_{1}=A_{s, p} \hat{H}_{2}, \\
A_{s, p}=2 \omega_{*}^{2}\left(\sin ^{2} \theta_{p}+\sin ^{2}\left(\theta_{s}+\theta_{p}\right)-2 \sin ^{2} \theta_{s}\right) \\
B_{s, p}=2 \sqrt{3} \omega_{*}^{2}\left(\sin ^{2} \theta_{p}-\sin ^{2}\left(\theta_{s}+\theta_{p}\right)\right) .
\end{gathered}
$$

Решая систему (38) относительно $\hat{H}_{1}, \hat{H}_{2}$ и применяя обратное преобразование Фурье, получим

$$
\begin{aligned}
& \operatorname{dev} \mathscr{H}=H_{1}(\mathbf{i i}-\mathbf{j} \mathbf{j})+H_{2}(\mathbf{i j}+\mathbf{j i}), \\
H_{1}= & \frac{1}{(2 N+1)^{2}} \\
& \times \sum_{s, p=-N}^{N} \frac{A_{s, p}^{2} \hat{H}_{1}^{0}+A_{s, p} B_{s, p} \hat{H}_{2}^{0}}{A_{s, p}^{2}+B_{s, p}^{2}} \cos \left(2 \theta_{s} k+2 \theta_{p} n\right), \\
H_{2}= & \frac{1}{(2 N+1)^{2}} \\
& \times \sum_{s, p=-N}^{N} \frac{A_{s, p} B_{s, p} \hat{H}_{1}^{0}+B_{s, p}^{2} \hat{H}_{2}^{0}}{A_{s, p}^{2}+B_{s, p}^{2}} \cos \left(2 \theta_{s} k+2 \theta_{p} n\right),
\end{aligned}
$$

где $\theta_{s}, \theta_{p}$ определены формулой (34). В термодинамическом пределе $(N \rightarrow \infty)$ суммы в формуле (39) превращаются в интегралы. Решение (39) получено при произвольных начальных условиях. Видно, что в общем случае $\operatorname{dev} \mathscr{H} \neq 0$; следовательно, энергия неравномерно распределяется по направлениям.

Рассмотрим случай, когда в начальный момент времени частицы имеют независимые случайные скорости и нулевые перемещения. При этом $\mathscr{H}_{0}=\mathscr{K}_{0} \delta_{k} \delta_{n}$, где $\mathscr{K}_{0}=\frac{m}{2}\left\langle\mathbf{v}_{i}^{0} \mathbf{v}_{i}^{0}\right\rangle$. Можно показать, что для бесконечного кристалла $(N \rightarrow \infty)$ выполняются тождества

$$
\begin{gathered}
\sum_{s, p=-N}^{N} \frac{A_{s, p}^{2}}{A_{s, p}^{2}+B_{s, p}^{2}}=\sum_{s, p=-N}^{N} \frac{B_{s, p}^{2}}{A_{s, p}^{2}+B_{s, p}^{2}}, \\
\sum_{s, p=-N}^{N} \frac{A_{s, p} B_{s, p}}{A_{s, p}^{2}+B_{s, p}^{2}}=0 .
\end{gathered}
$$

Подставляя $\mathscr{H}_{0}$ в решение (39) и используя тождества (40), получим при $k=n=0$

$$
\operatorname{dev} \mathscr{H}=\frac{1}{2} \operatorname{dev} \mathscr{K}_{0}, \operatorname{dev} \mathscr{K}=\frac{1}{4} \operatorname{dev} \mathscr{K}_{0} .
$$

Из (41) видно, что в гармонической треугольной решетке равного распределения кинетической энергии по направлениям не происходит. Если проекции начальных скоростей частиц на оси $x$ и $y$ не коррелируют, то из формул (41) следует простое выражение для компонент тензора температуры (13)

$$
T_{x x}-T_{y y}=\frac{1}{4}\left(T_{x x}^{0}-T_{y y}^{0}\right),
$$

где $T_{x x}=\mathbf{i} \cdot \mathscr{T} \cdot \mathbf{i}, T_{y y}=\mathbf{j} \cdot \mathscr{T} \cdot \mathbf{j} ; T_{x x}^{0}, T_{y y}^{0}$ - начальные значения $T_{x x}, T_{y y}$. Следовательно, тензор температуры (13), вообще говоря, не является шаровым даже в стационарном состоянии.

Рассмотрим ковариацию скоростей различных частиц в стационарном состоянии

$$
\left\langle\mathbf{v}(\mathbf{r}) \mathbf{v}\left(\mathbf{r}+k \mathbf{a}_{\alpha}\right)\right\rangle=\frac{2}{m} \mathscr{K}\left(k \mathbf{a}_{\alpha}\right) .
$$

В одномерном случае [17] данная ковариация равна нулю для всех $k>0$. В двумерном случае в силу формулы (22) равны нулю только шаровые части тензоров (43). Девиаторы тензоров (43), вообще говоря, отличны от нуля. Они вычисляются на основе формул (14), (35) и (39). Анализ формулы (39) показывает, что ковариация скоростей убывает обратно пропорционально квадрату расстояния между частицами. Кроме

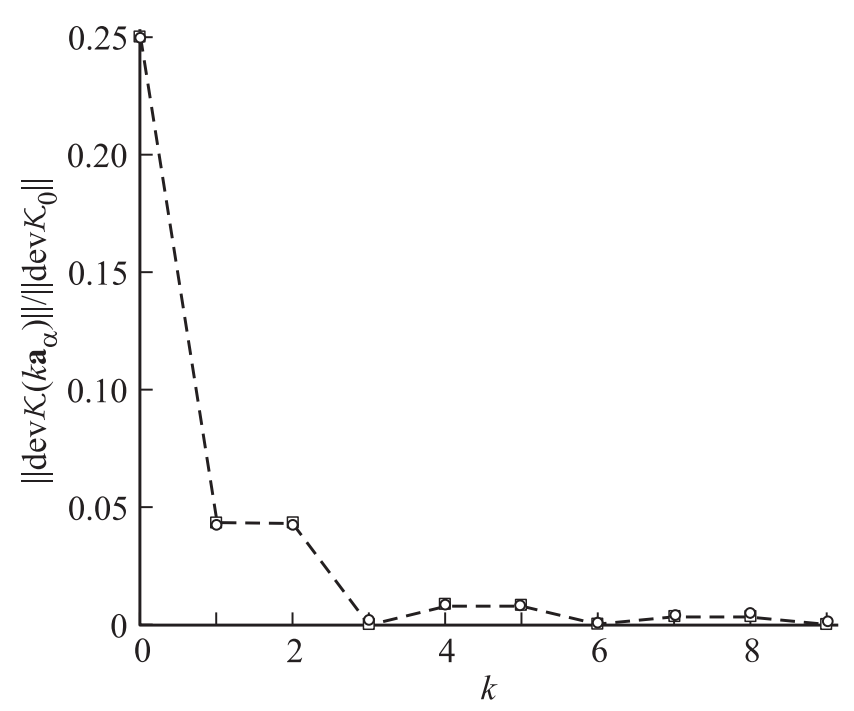

Рис. 2. Обобщенная кинетическая энергия в стационарном состоянии для треугольной решетки. Величина $\left\|\operatorname{dev} \mathscr{K}\left(k \mathbf{a}_{\alpha}\right)\right\|$ пропорциональна ковариации скоростей частиц, находящихся на расстоянии $k a, a-$ равновесное расстояние (см. (43)). Линия соединяет точки, полученные на основе формулы (39) при $N=150$, кружки и квадраты - численное решение уравнений динамики решетки (2) ( $\alpha=1$ и 2). 
того, норма ${ }^{13}\left\|\operatorname{dev} \mathscr{K}\left(k \mathbf{a}_{\alpha}\right)\right\|$ не зависит от направления $\alpha$.

Для проверки проведем сравнение аналитического решения (39) с результатами численного решения уравнений динамики решетки (2). Здесь и далее для численного решения используется метод Верле с шагом интегрирования $5 \cdot 10^{-3} \tau_{*}$, где $\tau_{*}=2 \pi / \omega_{*}$. Решение проводится при периодических граничных условиях. Ячейка периодичности содержит $10^{6}$ частиц. В начальный момент времени частицы имеют независимые случайные скорости, направленные вдоль одного из базисных векторов, и нулевые перемещения. Сравнение численного решения уравнений динамики решетки (2) с аналитическим решением (39) приведено на рис. 2. Видно, что на рисунке данные решения практически неотличимы.

Таким образом, полученное аналитическое решение показывает, что в гармонических кристаллах тензор температуры, вообще говоря, не является шаровым $\left(T_{x x} \neq T_{y y}\right)$. Кроме того, ковариация скоростей соседних частиц $\left\langle\mathbf{v}_{i} \mathbf{v}_{j}\right\rangle$ не равна нулю. Иными словами, скорости частиц не являются статистически независимыми.

8.3. Перераспределение энергии по пространственным направлениям. В разделе 6 показано, что перераспределение энергии по пространственным направлениям описывается уравнением (23) для $\operatorname{dev} \mathscr{H}$. В этом подразделе приводится решение данного уравнения для треугольной решетки.

Пусть в начальный момент времени частицы имеют независимые случайные скорости и нулевые перемещения. Ограничимся рассмотрением случая, когда компоненты скорости по направлениям і и $\mathbf{j}$ независимы. Тогда начальные условия примут вид

$$
\begin{gathered}
\operatorname{dev} \mathscr{\mathscr { H }}=\operatorname{dev} \mathscr{H}_{0}=H_{1}^{0}(\mathbf{i i}-\mathbf{j} \mathbf{j}) \delta_{k} \delta_{n}, \\
\dot{\mathscr{H}}=0, \quad \ddot{\mathscr{H}}=0, \quad \dddot{\mathscr{H}}=0 .
\end{gathered}
$$

Рассмотрим преобразование Фурье от уравнения (23)

$$
\begin{gathered}
\operatorname{dev} \dddot{\mathscr{\mathscr { H }}}-2(\hat{\mathscr{D}} \cdot \operatorname{dev} \ddot{\mathscr{\mathscr { H }}}+\operatorname{dev} \ddot{\mathscr{\mathscr { H }}} \cdot \hat{\mathscr{D}})+\hat{\mathscr{D}}^{2} \cdot \operatorname{dev} \hat{\mathscr{H}} \\
-2 \hat{\mathscr{D}} \cdot \operatorname{dev} \hat{\mathscr{H}} \cdot \hat{\mathscr{D}}+\operatorname{dev} \hat{\mathscr{H}} \cdot \hat{\mathscr{D}}^{2}=0, \\
\hat{\mathscr{D}}=-4 \omega_{*}^{2}\left(\sin ^{2} \theta_{s} \mathbf{e}_{1} \mathbf{e}_{1}+\sin ^{2} \theta_{p} \mathbf{e}_{2} \mathbf{e}_{2}+\sin ^{2}\left(\theta_{s}+\theta_{p}\right) \mathbf{e}_{3} \mathbf{e}_{3}\right) .
\end{gathered}
$$

Для тензора $\operatorname{dev} \hat{\mathscr{H}}$ будем использовать представление в декартовом базисе (37). Компоненты $\hat{H}_{1}, \hat{H}_{2}$ тензоpa $\operatorname{dev} \hat{\mathscr{H}}$ связаны первым уравнением системы (38). ${ }^{14}$ Умножая уравнения (45) на $\mathbf{i i}-\mathbf{j j}$ с учетом соотношения (38), получим уравнение для $\hat{H}_{1}$

$$
\dddot{\hat{H}}_{1}-2 \operatorname{tr} \hat{\mathscr{D}} \ddot{H}_{1}+\left(A_{s, p}^{2}+B_{s, p}^{2}\right) \hat{H}_{1}-A_{s, p}^{2} H_{1}^{0}=0,
$$

с соответствующими начальными условиями

$$
\hat{H}_{1}=H_{1}^{0}, \quad \dot{\hat{H}}_{1}=0, \ddot{\hat{H}}_{1}=0, \dddot{\hat{H}}_{1}=0,
$$

\footnotetext{
13 Здесь используется норма $\|\mathscr{X}\|=\mathscr{X} \cdot \mathscr{X}$.

14 Данная формула следует из закона сохранения (18).
}

где $A_{s, p}, B_{s, p}$ определены формулой (39). Решая уравнение (46) и применяя обратное преобразование Фурье, получим

$$
\begin{gathered}
H_{1}=\frac{H_{1}^{0}}{(2 N+1)^{2}} \sum_{s, p=-N}^{N} \frac{\cos \left(2 \theta_{s} k+2 \theta_{p} n\right)}{A_{s, p}^{2}+B_{s, p}^{2}} \\
\times\left(A_{s, p}^{2}+\frac{B_{s, p}^{2}}{\Omega_{+}^{2}-\Omega_{-}^{2}}\left(\Omega_{+}^{2} \cos \left(\Omega_{-} t\right)-\Omega_{-}^{2} \cos \left(\Omega_{+} t\right)\right)\right), \\
\Omega_{ \pm}=2 \omega_{*}\left(\sin ^{2} \theta_{s}+\sin ^{2} \theta_{p}\right. \\
\quad+\sin ^{2}\left(\theta_{s}+\theta_{p}\right) \pm \sqrt{3}\left(\sin ^{2} \theta_{s} \sin ^{2} \theta_{p}\right. \\
\left.\left.\quad+\left(\sin ^{2} \theta_{s}+\sin ^{2} \theta_{p}\right) \sin ^{2}\left(\theta_{s}+\theta_{p}\right)\right)^{1 / 2}\right)^{1 / 2}
\end{gathered}
$$

Аналогичное выражение для $H_{2}$ может быть получено с использованием первого уравнения системы (38). В частности, можно показать, что $H_{2}=0$ при $k=n=0$. В результате для $k=n=0$ имеем

$$
\begin{aligned}
& \operatorname{dev} \mathscr{H}=\operatorname{dev} \mathscr{H}_{0}\left[1-\frac{2}{(2 N+1)^{2}}\right. \\
& \left.\times \sum_{s, p=-N}^{N} \frac{B_{s, p}^{2}\left(\Omega_{+}^{2} \sin ^{2}\left(\frac{1}{2} \Omega_{-} t\right)-\Omega_{-}^{2} \sin ^{2}\left(\frac{1}{2} \Omega_{+} t\right)\right)}{\left(A_{s, p}^{2}+B_{s, p}^{2}\right)\left(\Omega_{+}^{2}-\Omega_{-}^{2}\right)}\right] .
\end{aligned}
$$

Сравнение аналитического решения (48) с численным решением уравнений динамики решетки (2) приведено

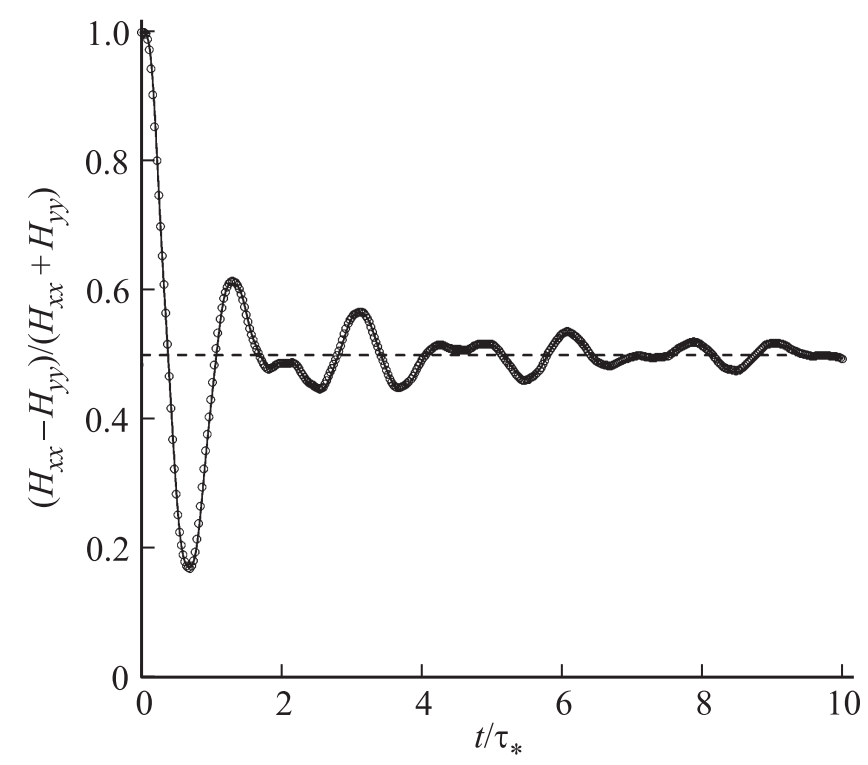

Рис. 3. Перераспределение энергий по пространственным направлениям в гармонической треугольной решетке. $H_{x x}, H_{y y}-$ компоненты тензора обобщенной полной энергии $\mathscr{H}\left(\mathbf{r}_{i}-\mathbf{r}_{j}\right)$ при $i=j$. Сплошная линия - аналитическое решение (48), точки - численное решение уравнений динамики решетки (2), штриховая линия - аналитическое решение (41) стационарной задачи. 
на рис. 3. В масштабе рисунка решения совпадают. Видно, что со временем разница между энергиями, соответствующими направлениям $x$ и $y$, уменьшается в 2 раза по сравнению с начальной. Данный результат совпадает с решением стационарной задачи (41), согласно которому $\operatorname{dev} \mathscr{H}=\frac{1}{2} \operatorname{dev} \mathscr{H}_{0}$.

Таким образом, процесс перераспределения энергии по пространственным направлениям в треугольной решетке со случайными начальными скоростями и нулевыми перемещениями описывается формулой (48). С течением времени система стремится к стационарному состоянию, в котором выполняются соотношения (41). Характерное время переходного процесса составляет величину порядка десяти периодов колебаний атомов $\tau_{*}$ (рис. 3).

8.4. Выравнивание кинетической и потенци альной энергй . Рассмотрим процесс выравнивания кинетической и потенциальной энергий. Выравнивание описывается уравнением (16) относительно обобщенного лагранжиана $\mathscr{L}$. Предположим, что в начальный момент времени частицы имеют независимые случайные скорости, равномерно распределенные по направлениям, и нулевые перемещения. Тогда начальные условия для уравнения (16) имеют вид

$$
\mathscr{L}=\frac{K_{0}}{2} \delta_{k} \delta_{n} Y, \dot{\mathscr{L}}=0, \ddot{\mathscr{L}}=4 \mathscr{D} \cdot \mathscr{L}, \ddot{\mathscr{L}}=0,
$$

где $K_{0}-$ начальное значение кинетической энергии, $\mathscr{Y}$ - единичный тензор. Для решения (16) воспользуемся предположением $\mathscr{D} \cdot \mathscr{L}=\mathscr{L} \cdot \mathscr{D}$. Тогда с учетом начальных условий (49) уравнение (16) приводится к виду

$$
\ddot{\mathscr{L}}=4 \mathscr{D} \cdot \mathscr{L} \text {. }
$$

Как видно, введенное предположение позволяет существенно упростить уравнение (16). Сравнение с численным решением уравнений динамики решетки (2) показывает, что уравнение (50) корректно описывает динамику лагранжиана $L=\left.\operatorname{tr} \mathscr{L}\right|_{i=j}$. Уравнение (50) равносильно системе трех независимых уравнений относитлеьно векторов $\mathbf{L}_{i}=\mathscr{L} \cdot \mathbf{e}_{i}$

$$
\ddot{\mathbf{L}}_{i}=4 \mathscr{D} \cdot \mathbf{L}_{i}, \quad i=1,2,3 .
$$

Начальные условия для $\mathbf{L}_{i}$ имеют вид

$$
\mathbf{L}_{i}=\frac{K_{0}}{2} \delta_{k} \delta_{n} \mathbf{e}_{i}, \dot{\mathbf{L}}_{i}=0
$$

Уравнение (51) с начальными условиями (52) имеет простую механическую аналогию. Задача о нахождении компонент $\mathbf{L}_{i}$ обобщенного лагранжиана эквивалентна задаче о колебаниях треугольной решетки, в которой в начальный момент времени одна частица имеет ненулевое перемещение, а скорости и перемещения остальных частиц равны нулю. Решение данной механической задачи приведено в Приложении. Пользуясь аналогией, для

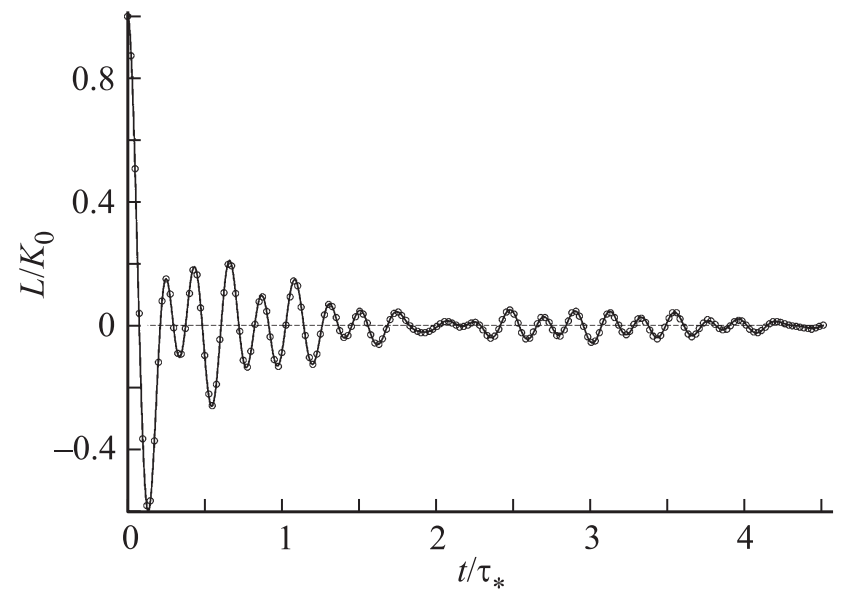

Рис. 4. Выравнивание кинетической и потенциальной энергий в гармонической треугольной решетке со случайными начальными скоростями. Линия - аналитическое решение (53), точки - численное решение уравнений динамики решетки (2).

лагранжиана $L=\left.\operatorname{tr} \mathscr{L}\right|_{i=j}$ получим

$$
\begin{aligned}
& L(t)=\frac{K_{0}}{2(2 N+1)^{2}} \sum_{s, p=-N}^{N}\left(\cos \left(2 \Omega_{1} t\right)+\cos \left(2 \Omega_{2} t\right)\right) \\
& \Omega_{j}^{4}-4 \omega_{*}^{2} \Omega_{j}^{2}\left(\sin ^{2} \theta_{s}+\sin ^{2} \theta_{p}+\sin ^{2}\left(\theta_{s}+\theta_{p}\right)\right) \\
& +12 \omega_{*}^{4}\left(\sin ^{2}\left(\theta_{s}+\theta_{p}\right) \sin ^{2} \theta_{s}+\sin ^{2}\left(\theta_{s}+\theta_{p}\right) \sin ^{2} \theta_{p}\right. \\
& \left.\quad+\sin ^{2} \theta_{s} \sin ^{2} \theta_{p}\right)=0, \quad j=1,2 .
\end{aligned}
$$

Вторая из формул (53) соответствует дисперсионному соотношению для треугольной решетки [36].

Для проверки формулы (53) проводилось численное решение уравнений динамики решетки (2). Для численного интегрирования использовался метод Верле с шагом интегрирования $\tau=10^{-3} \tau_{*}$. На рис. 4 показан процесс выравнивания кинетической и потенциальной энергий. Видно, что полученное аналитическое решение (53) в масштабе рисунка неотличимо от результатов численного решения уравнений динамики решетки.

Анализ формулы (53) показывает, что лагранжиан совершает затухающие колебания с амплитудой, обратно пропорциональной времени. В этом состоит существенное отличие от квадратной решетки, где лагранжиан затухает обратно пропорционально корню из времени (см. раздел 7). За время порядка $10 \tau_{*}$ разность между кинетической и потенциальной энергиями уменьшается на два порядка.

8.5. Вычисление ковариации перемещений в задачах о тепловом расширении. В работах $[37,38]$ показано, что ковариация перемещений играет важную роль при описании теплового расширения кристаллов. В частности, коэффициент теплового расширения для ангармонической треугольной решетки 
зависит от соотношения между компонентами следующего тензора в стационарном состоянии:

$$
\begin{aligned}
\mathscr{A} & =\left\langle\left(\mathbf{u}\left(\mathbf{r}+\mathbf{a}_{\alpha}\right)-\mathbf{u}(\mathbf{r})\right)\left(\mathbf{u}\left(\mathbf{r}+\mathbf{a}_{\alpha}\right)-\mathbf{u}(\mathbf{r})\right)\right\rangle \\
& =2 \xi(0)-\xi\left(\mathbf{a}_{\alpha}\right)-\xi\left(-\mathbf{a}_{\alpha}\right) .
\end{aligned}
$$

Без потери общности будем рассматривать случай $\alpha=1$. Тогда компоненты $A_{x x}=\mathbf{i} \cdot \mathcal{A} \cdot \mathbf{i}$ и $A_{y y}=\mathbf{j} \cdot \mathcal{A} \cdot \mathbf{j}$ тензора $\mathcal{A}$ в декартовом базисе (33) характеризуют продольные и поперечные деформации связи, вызванные тепловым движением частиц. В работе [38] показано, что коэффициент теплового расширения треугольной решетки существенно зависит от отношения $A_{y y} / A_{x x}$.

Вычислим тензор $\mathcal{A}$ в рамках рассматриваемой в настоящей работе модели гармонического кристалла. Для этого определим ковариацию перемещений на основе численного решения системы уравнений (7). Как и ранее уравнения решаются для ячейки периодичности в форме ромба (см. (33)). Используются следующие начальные условия

$$
\xi=0, \dot{\xi}=0, \kappa=\kappa_{0} \delta_{k} \delta_{n} \mathscr{Y}, \dot{\kappa}=0 .
$$

При этом величина $\kappa_{0}$ не влияет на интересующее нас отношение $A_{y y} / A_{x x}$. Для численного решения уравнений (7) используется алгоритм Верле с шагом по времени $10^{-3} \tau_{*}$. В результате решения получается следующее соотношение между компонентами тензора $\mathcal{A}$ :

$$
\frac{A_{y y}}{A_{x x}} \approx 1.43 \text {. }
$$

Формула (56) хорошо согласуется с результатами молекулярно-динамического моделирования, проведенного для кристалла Леннарда-Джонса в работе [38], где было получено $A_{y y} / A_{x x} \approx 1.435$.

Таким образом, используемая в настоящей работе модель гармонического кристалла может послужить для оценки соотношения между продольными и поперечными деформациями связей. Данная оценка необходима для вычисления коэффициента теплового расширения кристаллов в рамках подхода, изложенного в рабо$\operatorname{Tax}[37,38]$.

\section{9. Влияние нелинейности}

В данном разделе исследуется влияние малой нелинейности на два быстрых переходных процесса, описанных выше: 1) выравнивание кинетической и потенциальной энергий; 2) перераспределение энергии по пространственным направлениям.

Рассматривается треугольная решетка, частицы в которой взаимодействуют посредством потенциала Леннарда-Джонса:

$$
\Pi(r)=\varepsilon\left[\left(\frac{a}{r}\right)^{12}-2\left(\frac{a}{r}\right)^{6}\right],
$$

где $\varepsilon-$ энергия связи, $a-$ равновесное расстояние. Учитываются только взаимодействия ближайших

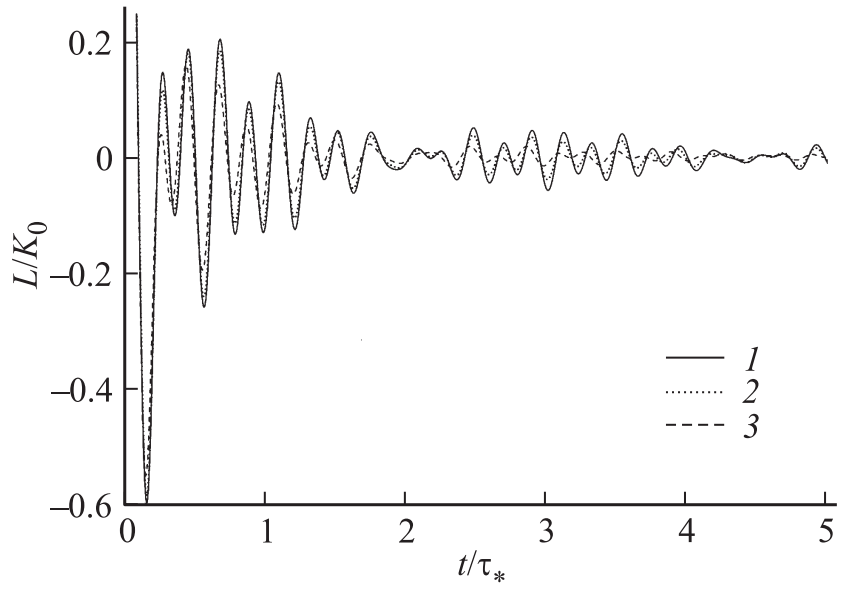

Рис. 5. Выравнивание кинетической и потенциальной энергий в треугольной решетке с взаимодействиями ЛеннардаДжонса. Частицы имеют случайные скорости, равномерно распределенные в круге радиуса $v_{0} . v_{0} / v_{d}=0.05(1), 0.25$ (2) и $0.5(3)$.

соседей. Моделирование проводится при периодических граничных условиях. В начальный момент времени частицам сообщаются независимые случайные скорости, равномерно распределенные в круге радиуса $v_{0}$. В качестве „масштаба““ скорости используется скорость диссоциации $v_{d}=\sqrt{2 \varepsilon / m}$. Начальные перемещения частиц равны нулю.

Варьируя амплитуду начальных скоростей частиц (температуру), можно изменять степень влияния нелинейности на поведение системы. Покажем, что при малых скоростях частиц (низких температурах) переходные тепловые процессы в кристалле Леннарда-Джонса хорошо описываются гармонической моделью.

Рассмотрим сначала влияние нелинейности на выравнивание кинетической и потенциальной энергий. Зависимость лагранжиана от времени, полученная в результате молекулярно-динамического моделирования, показана на рис. 5. Видно, что при переходе к стационарному состоянию кинетическая и потенциальная энергии выравниваются. При $v_{0}=0.05 v_{d}$ зависимость $L(t)$ в рассматриваемом временно́м интервале в пределах толщины линии совпадает с аналитическим решением для гармонического кристалла (53). При увеличении амплитуды начальных скоростей частиц влияние нелинейности приводит к тому, что кинетическая и потенциальная энергии выравниваются быстрее, чем в гармоническом кристалле.

Исследуем влияние нелинейности на процесс перераспределения энергии по пространственным направлениям. Пусть начальные скорости частиц направлены вдоль оси $x$, параллельной одному из базисных векторов решетки. В ходе молекулярно-динамического моделирования вычислялась разность компонент тензора температуры $T_{x x}, T_{y y}$, соответствующих направлениям $x$ и $y$. Зависимость $T_{x x}-T_{y y}$ от времени показана на 


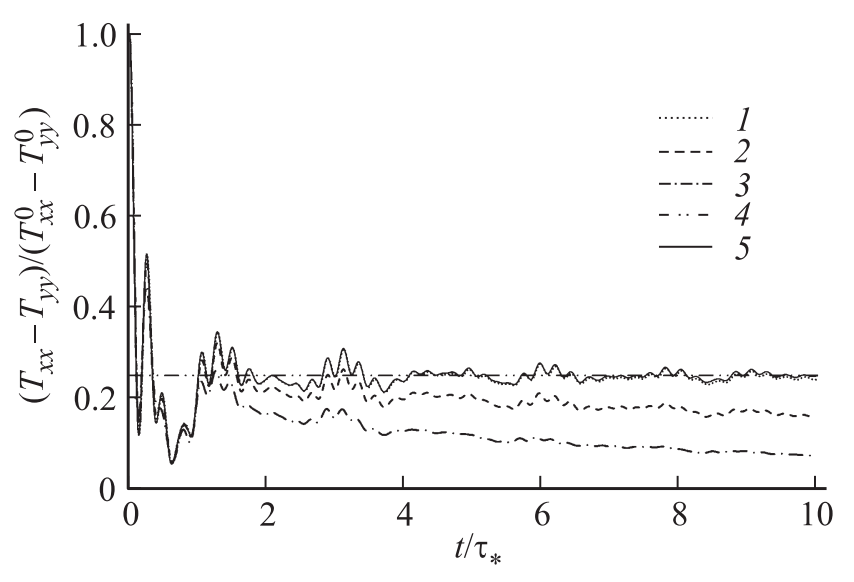

Рис. 6. Перераспределение кинетической энергии по пространственным направлениям в треугольной решетке с взаимодействиями Леннарда-Джонса. Частицы имеют случайные скорости, равномерно распределенные в круге радиуса $v_{0}$. $v_{0} / v_{d}=0.05(1), 0.25(2), 0.5(3) .4$ - аналитическое решение стационарной задачи для гармонического кристалла, 5 - численное решение уравнений динамики гармонического кристалла (2).

рис. 6. Приведенные кривые соответствуют среднему по 25 реализациям с различными случайными начальными скоростями. Из рисунка видно, что при переходе к стационарному состоянию разность $T_{x x}-T_{y y}$ в течение одного периода $\tau_{*}$ уменьшается примерно в 4 раза, после чего сравнительно медленно стремится к нулю.

Таким образом, переход к стационарному состоянию в слабо нелинейных кристаллах имеет два временны́х масштаба. На малых временах (порядка нескольких периодов $\tau_{*}$ ) переходные процессы хорошо описываются гармонической моделью. На больших временах $\left(t \gg \tau_{*}\right)$ добавляется новый эволюционный процесс, связанный с нелинейностью. В частности, нелинейность приводит к тому, что со временем кинетические энергии, соответствующие движению частиц в различных направлениях, выравниваются. Скорость эволюционного процесса зависит от величины нелинейности (температуры).

\section{0. Заключение}

В работе предложено аналитическое описание двух переходных тепловых процессов, происходящих в однородно нагретых гармонических кристаллах: 1) выравнивания кинетической и потенциальной энергий; 2) перераспределения энергии по пространственным направлениям. Получена замкнутая система уравнений с детерминированными начальными условиями, описывающая оба процесса в двухмерном и трехмерном случаях. Показано, что при переходе к стационарному состоянию кинетическая и потенциальная энергии вы- равниваются. Кинетическая энергия перераспределяется по пространственным направлениям, но теорема о равном распределении, вообще говоря, не выполняется. Иными словами, кинетическая температура проявляет тензорные свойства. Выведена система уравнений, позволяющая, в частности, определить связь тензора температуры в стационарном состоянии с начальными условиями.

В качестве примеров рассмотрены квадратная и треугольная решетки. В квадратной решетке перераспределения энергии по направлениям не происходит. Выравнивание кинетической и потенциальной энергий, соответствующих одному направлению, описывается функцией Бесселя. В треугольной решетке перераспределение энергии по направлениям происходит. Однако теорема о равном распределении, вообще говоря, не выполняется. Кроме того, ковариация скоростей пары частиц $\left\langle\mathbf{v}_{i} \mathbf{v}_{j}\right\rangle$ в стационарном состоянии, вообще говоря, не равна нулю. Она убывает обратно пропорционально квадрату расстояния между частицами. При переходе к стационарному состоянию разность между кинетической и потенциальной энергиями совершает затухающие колебания с амплитудой, обратно пропорциональной времени. В квадратной решетке аналогичные колебания затухают обратно пропорционально корню из времени. Характерное время данных переходных процессов для треугольной решетки составляет величину порядка десяти периодов колебаний атомов.

Численно исследовано влияние нелинейности на процесс перехода к стационарному состоянию. Показано, что малая нелинейность приводит к тому, что на рассмотренные быстрые процессы накладывается медленный процесс, вызванный наличием нелинейности. Таким образом, изложенные выше результаты применимы в том числе и для описания быстрых переходных процессов в слабо нелинейных системах.

Авторы благодарны М.Б. Бабенкову, Е.А. Ивановой, Д.А. Индейцеву, Н.Ф. Морозову и У.Г. Хуверу за полезные обсуждения.

\section{Приложение. Колебания треугольной решетки}

Рассмотрим треугольную решетку, состоящую из частиц массы $m$, соединенных пружинками жесткости $4 C$. Будем использовать периодические граничные условия. Ячейка периодичности имеет форму ромба со стороной, состоящей из $2 N+1$ частиц. Радиус-векторы частиц задаются формулой (33). Уравнения движения имеют вид

$$
\ddot{\mathbf{u}}_{k, n}=4 \mathscr{D} \cdot \mathbf{u}_{k, n} .
$$

В начальный момент времени центральная частица $(k=0, n=0)$ смещена вдоль связи е 1 на $u_{0}$. Начальные скорости и перемещения остальных частиц равны нулю. 
Введем новые переменные

$$
\begin{gathered}
\mathbf{u}_{k, n}=w_{1} \mathbf{e}_{1}+w_{2} \mathbf{e}_{2}, w_{1}=\frac{2}{3}\left(2 u_{1}+u_{2}\right), \\
w_{2}=\frac{2}{3}\left(2 u_{2}+u_{1}\right), \quad u_{i}=\mathbf{u}_{k, n} \cdot \mathbf{e}_{i}
\end{gathered}
$$

где векторы $\mathbf{e}_{i}$ определяются формулами (33).

Здесь и далее индексы $k, n$ у величин $w_{1}, w_{2}, u_{1}, u_{2}$ для краткости опущены. С учетом (П2) уравнения движения принимают вид

$$
\begin{gathered}
\ddot{w}_{1}=2 \omega_{*}^{2}\left(\Delta_{1}^{2}\left(2 w_{1}-w_{2}\right)+\Delta_{3}^{2}\left(w_{1}+w_{2}\right)\right), \\
\ddot{w}_{2}=2 \omega_{*}^{2}\left(\Delta_{2}^{2}\left(2 w_{2}-w_{1}\right)+\Delta_{3}^{2}\left(w_{1}+w_{2}\right)\right) .
\end{gathered}
$$

Начальные условия имеют вид

$$
\mathbf{u}_{k, n}=u_{0} \delta_{k} \delta_{n} \mathbf{e}_{1}, \quad w_{1}=u_{0} \delta_{k} \delta_{n}, \quad w_{2}=0, \quad \dot{w}_{1}=\dot{w}_{2}=0
$$

Применим дискретное преобразование Фурье (34) по индексам $k, n$ к уравнениям (П3). Для этого воспользуемся тождествами

$$
\begin{gathered}
\Phi\left(\Delta_{1}^{2} g_{k, n}\right)=-4 \sin ^{2} \theta_{s} \hat{g}_{s, p}, \quad \Phi\left(\Delta_{2}^{2} g_{k, n}\right)=-4 \sin ^{2} \theta_{p} \hat{g}_{s, p}, \\
\Phi\left(\Delta_{3}^{2} g_{k, n}\right)=-4 \sin ^{2}\left(\theta_{s}+\theta_{p}\right) \hat{g}_{s, p} \\
\hat{g}_{s, p}=\Phi\left(g_{k, n}\right), \quad \theta_{s}=\frac{\pi s}{2 N+1} .
\end{gathered}
$$

В результате для Фурье-образов $\hat{w}_{i}=\Phi\left(w_{i}\right)$ имеем

$$
\begin{aligned}
\ddot{\tilde{w}}_{1}= & -8 \omega_{*}^{2}\left[\left(2 \sin ^{2} \theta_{s}+\sin ^{2}\left(\theta_{s}+\theta_{p}\right)\right) \hat{w}_{1}\right. \\
& \left.+\left(\sin ^{2}\left(\theta_{s}+\theta_{p}\right)-\sin ^{2} \theta_{s}\right) \hat{w}_{2}\right], \\
\ddot{\tilde{w}}_{2}= & -8 \omega_{*}^{2}\left[\left(\sin ^{2}\left(\theta_{s}+\theta_{p}\right)-\sin ^{2} \theta_{p}\right) \hat{w}_{1}\right. \\
& \left.+\left(2 \sin ^{2} \theta_{p}+\sin ^{2}\left(\theta_{s}+\theta_{p}\right)\right) \hat{w}_{2}\right] .
\end{aligned}
$$

Собственные частоты $\Omega_{1}, \Omega_{2}$, соответствующие системе (П6), определяются уравнением

$$
\begin{aligned}
\Omega_{j}^{4} & -16 \omega_{*}^{2} \Omega_{j}^{2}\left(\sin ^{2} \theta_{s}+\sin ^{2} \theta_{p}+\sin ^{2}\left(\theta_{s}+\theta_{p}\right)\right) \\
& +192 \omega_{*}^{4}\left(\sin ^{2}\left(\theta_{s}+\theta_{p}\right) \sin ^{2} \theta_{s}+\sin ^{2}\left(\theta_{s}+\theta_{p}\right) \sin ^{2} \theta_{p}\right. \\
& \left.+\sin ^{2} \theta_{s} \sin ^{2} \theta_{p}\right)=0, j=1,2
\end{aligned}
$$

Начальные условия для $\hat{w}_{1}, \hat{w}_{2}$ имеют вид

$$
\hat{w}_{1}=u_{0}, \quad \hat{w}_{2}=0, \quad \dot{\hat{w}}_{1}=\dot{\hat{w}}_{2}=0 .
$$

Решая систему (П6) с начальными условиями (П8) и применяя обратное преобразование Фурье, получим

$$
\mathbf{u}_{k, n}=w_{1} \mathbf{e}_{1}+w_{2} \mathbf{e}_{2},
$$

$$
\begin{aligned}
w_{1}= & \frac{u_{0}}{2(2 N+1)^{2}} \\
& \times \sum_{s, p=-N}^{N} \cos \left(2 \theta_{s} k+2 \theta_{p} n\right)\left(\cos \left(\Omega_{1} t\right)+\cos \left(\Omega_{2} t\right)\right. \\
& \left.+16 \omega_{*}^{2} \frac{\sin ^{2} \theta_{s}-\sin ^{2} \theta_{p}}{\Omega_{1}^{2}-\Omega_{2}^{2}}\left(\cos \left(\Omega_{1} t\right)-\cos \left(\Omega_{2} t\right)\right)\right), \\
w_{2}= & \frac{8 u_{0}}{(2 N+1)^{2}} \sum_{s, p=-N}^{N} \frac{\omega_{*}^{2}\left(\sin ^{2}\left(\theta_{s}+\theta_{p}\right)-\sin ^{2} \theta_{p}\right)}{\Omega_{1}^{2}-\Omega_{2}^{2}} \\
& \times\left(\cos \left(\Omega_{1} t\right)-\cos \left(\Omega_{2} t\right)\right) \cos \left(2 \theta_{s} k+2 \theta_{p} n\right) .
\end{aligned}
$$

Формулы (П9) дают точное решение задачи о колебаниях треугольной решетки, в которой одна из частиц имеет начальное смещение.

\section{Список литературы}

[1] Р.В. Гольдштейн, Н.Ф. Морозов. Физ. мезомеханика 10, 5, 17 (2007).

[2] I.F. Golovnev, E.I. Golovneva, V.M. Fomin. Comp. Mater. Sci. 36, 176 (2006).

[3] S.N. Korobeynikov, V.V. Alyokhin, B.D. Annin, A.V. Babichev. Arch. Mech. 64, 367 (2012).

[4] J.C. Reid, D.J. Evans, D.J. Searles. J. Chem. Phys. 136, 02110 (2012).

[5] C.F. Petersen, D.J. Evans, S.R. Williams. J. Chem. Phys. 144, 074107 (2016).

[6] Г.И. Канель, С.В. Разоренов, А.В. Уткин, В.Е. Фортов. Ударноволновые явления в конденсированных средах. Янус-К, М. (1996). 407 с.

[7] B.L. Holian, W.G. Hoover, B. Moran, G.K. Straub. Phys. Rev. A 22, 2798 (1980).

[8] B.L. Holian, M. Mareschal. Phys. Rev. E 82, 026707 (2010).

[9] W.G. Hoover, C.G. Hoover, K.P. Travis. Phys. Rev. Lett. 112, 144504 (2014).

[10] F. Silva, S.M. Teichmann, S.L. Cousin, M. Hemmer, J. Biegert. Nater. Commun. 6, 6611 (2015).

[11] С.И. Ашитков, П.С. Комаров, М.Б. Агранат, Г.И. Канель, В.Е. Фортов. Письма в ЖЭТФ 98, 439 (2013).

[12] N.A. Inogamov, Yu.V. Petrov, V.V. Zhakhovsky, V.A. Khokhlov, B.J. Demaske, S.I. Ashitkov, K.V. Khishchenko, K.P. Migdal, M.B. Agranat, S.I. Anisimov, V.E. Fortov, I.I. Oleynik. AIP Conf. Proc. 1464, 593 (2012).

[13] K.V. Poletkin, G.G. Gurzadyan, J. Shang, V. Kulish. Appl. Phys. B 107, 137 (2012).

[14] D.A. Indeitsev, V.N. Naumov, B.N. Semenov, A.K. Belyaev. ZAMM 89, 279 (2009).

[15] В.А. Кузькин, А.М. Кривцов. ДАН 472, 5, 529 (2017).

[16] Z. Rieder, J.L. Lebowitz, E. Lieb. J. Math. Phys. 8, 1073 (1967).

[17] А.М. Кривцов. ДАН 458, 279 (2014). 
[18] A.M. Krivtsov. ArXiv:1509.02506 [cond-mat.stat-mech] (2015).

[19] А.М. Кривцов. ДАН 464, 162 (2015).

[20] T.V. Dudnikova, A.I. Komech, H. Spohn. J. Math. Phys. 44, 2596 (2003).

[21] T.V. Dudnikova, A.I. Komech, N.J. Mauser. J. Stat. Phys. 114, 1035 (2004).

[22] D.J. Evans, D.J. Searles, S.R. Williams. J. Stat. Mech. 2009, P07029 (2009).

[23] H. Spohn, J.L. Lebowitz. Commun. Math. Phys. 54, 97 (1977).

[24] A. Dhar, R. Dandekar. Physica A 418, 49 (2014).

[25] А.В. Савин, О.В. Гендельман. ФТТ 43, 341 (2001).

[26] S. Lepri, R. Livi, A. Politi. Phys. Rep. 377, 1 (2003).

[27] M. Jara, T. Komorowski, S. Olla. Commun. Math. Phys. 339, 407 (2015).

[28] М.Б. Бабенков, А.М. Кривцов, Д.В. Цветков. Физ. мезомеханика 19, 1, 60 (2016).

[29] А.И. Михайлин, Л.В. Жигилей, А.И. Слуцкер. ФТТ 37, 1784 (1995).

[30] А.И. Слуцкер. ФТТ 46, 1606 (2004).

[31] Л.Д. Ландау, Е.М. Лифшиц. Теоретическая физика. Статистическая физика. Наука, М. (1976). 584 с.

[32] А.М. Кривцов. В сб.: Вопросы математической физики и прикладной математики. Материалы семинара, приуроченного к 75-летию проф. Э.А. Троппа. ФТИ им. А.Ф. Иоффе, СПб (2016). С. 63-81.

[33] W.G. Hoover, Computational statistical mechanics. Elsevier, N.Y. (1991). 330 p.

[34] G.S. Mishuris, A.B. Movchan, L.I. Slepyan. J. Mech. Phys. Solids 57, 1958 (2009).

[35] V.A. Kuzkin, A.M. Krivtsov, E.A. Podolskaya, M.L. Kachanov. Phil. Mag. 96, 1538 (2016).

[36] P. Dean. Proc. Camb. Phil. Soc. 59, 383 (1963).

[37] А.М. Кривцов, В.А. Кузькин. МТТ 3, 67 (2011).

[38] А.Ю. Панченко, Е.А. Подольская, А.М. Кривцов. ДАН 473, 2 (2017). 